\title{
Research on the Diagnosis and Treatment of Pediatric Gastrointestinal Dysfunction
}

\author{
Yongchao $\mathrm{Li}^{1, \mathrm{a}}$, Dan Wang ${ }^{2, \mathrm{~b}}$, Zhenbo Shu ${ }^{3, \mathrm{c} \text { Correspondent Author }}$ \\ ${ }^{1,3}$ Department of Gastrointestinal Colorectal and Anal Surgery, China-Japan Union Hospital, Jilin \\ University, Changchun, 130033 \\ ${ }^{2}$ Operation Room of China-Japan Union Hospital, Jilin University, Changchun, 130033 \\ aemail, bemail, ${ }^{\mathrm{c} e m a i l,}$
}

\section{Keywords: Diagnosis and Treatment, Pediatric Gastrointestinal Dysfunction}

\begin{abstract}
Gastrointestinal dysfunction is a multi-organ failure dysfunction syndrome, and gastrointestinal injury is the cause of organ failure caused by the River. Clinical symptoms in patients with gastrointestinal failure occurs, treatment more difficult, have a higher mortality rate, more difficult to treat a class of diseases. In addition to the primary disease treatment outside of gastrointestinal dysfunction in children include intervention measures can effectively reduce the mortality rate of children.
\end{abstract}

\section{Introduction}

Gastrointestinal digestion and absorption function of vital organs is completed. In recent years, due to the in-depth study of a single organ failure developed to multi-system organ failure (MSOF), subjected to systemic inflammatory response syndrome (SIRS) and multiple organ dysfunction syndrome (MODS) stage. Gastrointestinal dysfunction is not only one of the target organ MODS, and was the cradle stimulate inflammation, known as multiple organ failure of the engine. When the rescue critically ill children, children with gastrointestinal dysfunction and failure is considered to be under stress MSOF of "Origins. Therefore, the treatment of children with gastrointestinal dysfunction is an important issue in clinical pediatrics.

\section{The Concept of Pediatric Gastrointestinal Dysfunction}

Gastrointestinal dysfunction or failure as an independent diagnostic terms, not by much clinical use, it is not like heart failure, renal failure, respiratory failure as familiar and attention to the usual with "toxic intestinal paralysis" or "should bowel ulcer "and other diagnostics to be summed up, in fact, when the body hit (stress) occurs after ischemia and hypoxia, intestinal dysfunction, a large number of bacterial translocation, endotoxemia aggravating make disease worse, children with abdominal distension, bowel sounds diminished or disappeared and gastrointestinal bleeding, known as gastrointestinal dysfunction, and gastrointestinal failure means the stress ulcer or blood transfusion appears highly toxic intestinal paralysis abdominal distension.

Healthy flora in the gastrointestinal tract to maintain the ecological balance, when the body suffered after being hit pathogenic factors causing microcirculation, redistribution of systemic blood, stomach showed hypoperfusion, gastrointestinal tract are the first to suffer from hypoxic-ischemic damage organ, it is said, "the gastrointestinal tract is MSOF initiating organ." Since a large number of bacteria in the gastrointestinal tract, severe infection, hypoxemia and extensive use of broad-spectrum antibiotics, long-term fasting, gastrointestinal stress ulcer bleeding and other factors alter the intestinal barrier, intestinal immune suppression, intestinal bacteria shift, so that bacterial toxins and inflammatory mediators continued into the blood and lymph cause second strike, starting and accelerating the development of MODS (2). The importance of gastrointestinal dysfunction or failure in critically ill patients in the development process instructions. Literature relevant domestic MSOF of content related to gastrointestinal function only $30 \%$ to $40 \%$ (3), indicating that the majority of clinicians to gastrointestinal dysfunction lack of understanding of the concept, not much 
clinical experience that misdiagnosis of chances more tips to strengthen continuing education, awareness, diagnosis is necessary.

\section{Diagnosis of Gastrointestinal Dysfunction in Children}

That bloating abdominal distension. Because the intestine flatulence, intestinal autonomic dysfunction make digestive dysfunction due to other reasons; systemic infection, pathological state sepsis, shock, respiratory failure, microcirculation and blood redistribution, so that expansion of gastrointestinal ischemia weakness and abdominal distention; peritonitis, resulting in intestinal malabsorption can lead to paralysis gas abdominal injury. Bloating is a clinical symptoms, often above the xiphoid, if sustained abdominal distension is not flat and there is increased tension can be considered pathological, often accompanied with severe acute tolerance and symptoms of poisoning, when paralytic ileus with abdominal pain, vomiting, and not exhaust defecation, bowel sounds diminished or disappeared. Critically ill children bloating is often a sign of disease progression and irreversible. I observed hospital ICU patients showed that bloating occurs in infancy, accounting for $88.43 \%$, primary pathogenesis of infectious diseases of $78.51 \%$, prone when accompanied by malnutrition and other underlying diseases; bloating with spit coffee-like liquid accounted for 47.93 percent, with bowel sounds diminished or disappeared accounted for 38.02\%; bloating occur before organ disorders average number of 1.92, respiratory system disorders and brain edema were more common, the development of disease progression appear to 3.41 organ dysfunction after abdominal distension, microcirculation, renal dysfunction and pulmonary hemorrhage increased significantly. Also observed that $60 \%$ of critically ill children with severe abdominal distension appear successor of circulatory failure, and $71.7 \%$ of children die within $48 \mathrm{~h}$. Description gut as irreversible shock "hub" organ poor prognosis. When bloating monitoring of serum electrolytes, the presence of only 19.83\% hypokalemia, often accompanied by high blood sugar and elevated blood urea nitrogen, some children with significant acidosis, hypoxemia. Children in the event of abdominal distension, should be fully ruled out mechanical intestinal obstruction, intestinal perforation, surgical abdomen, orthostatic X-ray film to understand whether the flatulence, liquid gas plane or free gas below the diaphragm.

2 is the body's stress ulcer severe stress. Especially when gastrointestinal mucosal lesions occur in acute severe trauma, burns, shock and systemic infection and so on. Gastrointestinal ischemia, energy metabolism and mucosal damage is an important defense mechanism causes of stress ulcer. Gastric mucosal injury is an important factor, mucus - bicarbonate $\mathrm{H}+$ reverse diffusion barrier defense to maintain the $\mathrm{pH}$ gradient within the mucosa, bile reflux and gastric mucosal defense mechanisms resulting in the role of free radicals damage, ischemia and hypoxia plus damaging factors caused stress ulcer. Lesions were located in the gastric fundus and body, first appeared pale dot ischemic area, happen soon congestion, edema and hemorrhage point sheet, even superficial erosion and concurrent gastrointestinal bleeding, severe and extended to the duodenum entire gastrointestinal mucosa and caused perforation. Early clinical manifestations are often not very clear, few children may be varying degrees of abdominal distention, abdominal pain, nausea, severe overshadowed by the primary disease digestive symptoms often appear black (tarry), the occurrence of sudden vomiting or vomit coffee-like stomach contents as an early manifestation. Fiber endoscopy is the main method of early diagnosis, selective angiography shows contrast extravasation into a ball next to accumulate in the blood vessels and eventually becoming dissipated, X-ray film, see Tips ulcer when free intraperitoneal gas, stomach ultrasound image may have increased thick mucosal folds hypertrophy. Out of coffee-like substance and fecal occult blood test was positive in the stomach is a simple laboratory examinations early indicators.

In acute critical illness or sudden state of the gradual emergence of severe abdominal distension, bowel sounds decreased or disappeared, spit coffee-like substance or blood in the stool, may consider gastrointestinal dysfunction. Fry (1991 Nian) bloating in MODS/MSOF diagnostic criteria and can not tolerate food by mouth more than five days called gastrointestinal dysfunction, said the emergence of stress ulcer blood transfusion gastrointestinal failure. National Critical Care Conference (1995) in staging and MODS illness severity score, the abdominal bloating, decreased 
bowel sounds recorded 1 min, height abdominal bloating, bowel sounds nearly disappeared in mind two points, paralytic ileus stress ulcer bleeding or three points. Pediatric critical illness score (1996) as one of the Gastrointestinal Dysfunction Score of 10, appears recorded 6 points of stress ulcer, stress ulcer with intestinal paralysis in mind four points, the stress ulcer bleeding blood transfusion and who appears highly toxic intestinal paralysis bloating gastrointestinal dysfunction in children as standard.

When the diagnosis of gastrointestinal dysfunction should note the following: Understanding the primary disease, more severe infections, ischemia and hypoxia, shock or trauma, surgery on the basis of acute critical illness occurs; timely exclude themselves gastrointestinal diseases and acute surgical abdomen disease, such as necrotizing enterocolitis, mechanical intestinal obstruction, intestinal perforation, hemorrhage, ascites; closely monitoring the state of other organs, gastrointestinal dysfunction is often MODS/MSOF part; attention to body condition and internal environment monitoring, an overall estimate of the disease.

\section{Treatment of Children with Gastrointestinal Dysfunction}

Early diagnosis and timely treatment is the key to a comprehensive treatment of gastrointestinal dysfunction. Early treatment may suspend disease development, access to better treatment. Common treatments include: the removal of the lesion, infection control, protect the gastrointestinal mucosa barrier function, gastrointestinal bleeding to stop, eliminate bloating. Enhancing peristalsis, controlled release of inflammatory mediators. Strengthen support for therapy.

Cause of treatment: positive control is the basis of the primary disease treatment to correct dysfunction of various systems and organs to protect vital organs function, improve circulation. Infection control and removal of the lesion, a reasonable choice of antibiotics, fluid therapy and good heat supply.

Relieve bloating: abdominal distension critically ill children is often a sign of disease progression and irreversible. According to reports, bloating occurs in early childhood, accounting for $88.43 \%$, primary pathogenesis of infectious diseases of $78.51 \%$ was also observed in $60 \%$ of critically ill children with severe abdominal distension appear successor of circulatory failure, and in $71.7 \%$ of children within $48 \mathrm{~h}$ death, described as intestinal irreversible shock "hub" organ poor prognosis. Treatment: fasting; and bloating worse after eating or gastric retention and upper gastrointestinal bleeding should be fasting bloating persists, promptly after feeding until symptoms improved; ${ }^{\circ}$ decompression can reduce the accumulating gases swallowing, digestion aspiration Gyeonggi retention of liquids and gases to reduce pressure within the gastrointestinal tract, can also be found in the stomach of coffee-like liquid as soon as possible; intestine exhaust or 5\% saline 20 $\sim 50 \mathrm{ml}$ enema to stimulate peristalsis knot; $1 / 4$ attention to potassium; $1 / 2$ applications neostigmine Ming cholinesterase inhibition, increases intestinal peristalsis, promote exhaust; apply phentolamine improve lung ventilation, gastrointestinal smooth muscle excitability increased intestinal creeping reduce bloating; acupuncture (Zusanli, gu, abdominal, etc.) or umbilical dressing (light blue or mustard), can stimulate the nerve endings, promote bowel movements.

Stress ulcer: early clinical manifestations are often not very clear, few children may be varying degrees of abdominal distention, abdominal pain, nausea, severe overshadowed by the primary disease digestive symptoms often appear black stools, vomiting or sudden spit coffee-like material for the early performance. Fiber endoscopy is the main method of early diagnosis. When fasting before treatment using gastric lavage with cold saline or $1.4 \%$ sodium bicarbonate, with its cimetidine every $10 \sim 20 \mathrm{mg} / \mathrm{kg}$ injected into the stomach to retain a good hemostatic effect, the effective rate of $87 \%$ ( 6), usually 1 or 2 times can be adequately controlled. While giving the drugs to stop bleeding and other conservative treatment, surgical treatment if necessary.

Bacterial translocation and selective digestive decontamination technique: selective digestive decontamination operation, improve the intestinal micro-ecological environment, ecological in antibiotic therapy, the choice of the majority of potential pathogens sensitive to obligate tired almost no activity of bacteria, oral easily absorbed, there is a certain inhibit or kill candida and other antibiotics such as norfloxacin, co-trimoxazole, amphotericin B and the like. Treatment is generally 
one week.

Protect the gastrointestinal mucosa barrier function, prevention of endogenous infection within (7). Intestinal tract is known as multiple organ dysfunction start actuator, when structural damage intestinal mucosal barrier by invasive bacterial translocation and endotoxin stimulate systemic inflammatory response, leading to sepsis and MODS. Give VitC, VitE and other free radical scavengers to correct persistent hypoperfusion comprehensive treatment measures.

TCM treatment: Chinese medicine, pediatric gastrointestinal dysfunction occurs with children "spleen often insufficient" relevant (6). Integrative Medicine gastrointestinal dysfunction has a good effect $(9,10)$. Chinese Herbs and feeding: Zheng Jian abdominal distention, poor stool, scarlet tongue, tongue thick and greasy, slippery pulse, hot repressor, gas-poor, should be heat dredge qi Xiaozhang, party election woody Penang pill: wood, betel nut, peel, orange peel, Curcuma, berberine, Cork, rhubarb, sweet. Zheng Jian abdominal distention, loose stools does not stink, vomit clear saliva, tongue fat, pale, white moss and moist, pulse Shen fine, serving cold evil block, qi poor, Expelling warm BOC gas, in addition to dampness full, warm in the party election Magnolia Decoction: Magnolia, ginger, orange peel, red Poria, grass cardamom, woody, licorice enema therapy: available said Jianshui oral medicine retention enema to promote gastrointestinal motility, reduce cavity distention conducive to bacterial endotoxin and discharged.

Salvia intravenous injection of phentolamine in combination therapy toxic intestinal paralysis, can reduce bloating, improve microcirculation. In short, gastrointestinal dysfunction participation MODS/MSOF pathological process, and its occurrence, development and prognosis have important implications sepsis gut reaction is that many inflammatory mediators "generator", leading to MODS/MSOF of occur. SIRS and MODS is a pediatric intensive care unit in a common disease, mortality more. SIRS or MODS progress to show the presence of gastrointestinal dysfunction before organ failure, should pay close attention and early intervention.

\section{Conclusion}

Gastrointestinal dysfunction after early detection and timely treatment can get good results, so early diagnosis is very important. This study, we use the combined modality therapy, good therapeutic effect, drugs are able to effectively improve gastrointestinal dysfunction in children, intestinal microcirculation and play a regulating effect, the majority of children do not appear in the study organ damage, the condition improved and it is the ideal treatment.

\section{References}

[1] Hu Haofu. Gastrointestinal dysfunction in children with multiple organ dysfunction syndrome status Chinese Journal of Pediatrics, 2000; 16 (1): 10.

[2] Tang Dinghua, Zhang Yuming, Zhang Guoqin. Pediatric wards strengthen clinical systemic inflammatory response syndrome and multiple organ dysfunction syndrome analysis • Pediatrics 1998; 36 (10): 585.

[3] Zhao Xiangwen. Pediatric gastrointestinal dysfunction Overview Chinese Journal of Pediatrics, 2000; 16 (1): 1.

[4] Zhu Yimin. Diagnosis and treatment of pediatric gastrointestinal dysfunction Chinese Journal of Pediatrics, 2001; 16 (1): 6.

[5] Zhu Yimin, Zhao Xiangwen. When critically ill circulatory failure and gastrointestinal disorders correlation of power (46 cases) Pediatric Emergency Medicine, 1996; 3 (2): 68. 